\title{
UNA ENTREVISTA CON EL ARQUITECTO DICKEN CASTRO DUQUE'
}

Jorge Alberto Villamizar Hernández *

El arquitecto Dicken Castro en su biblioteca Fuente: Jorge Alberto Villamizar Hernández

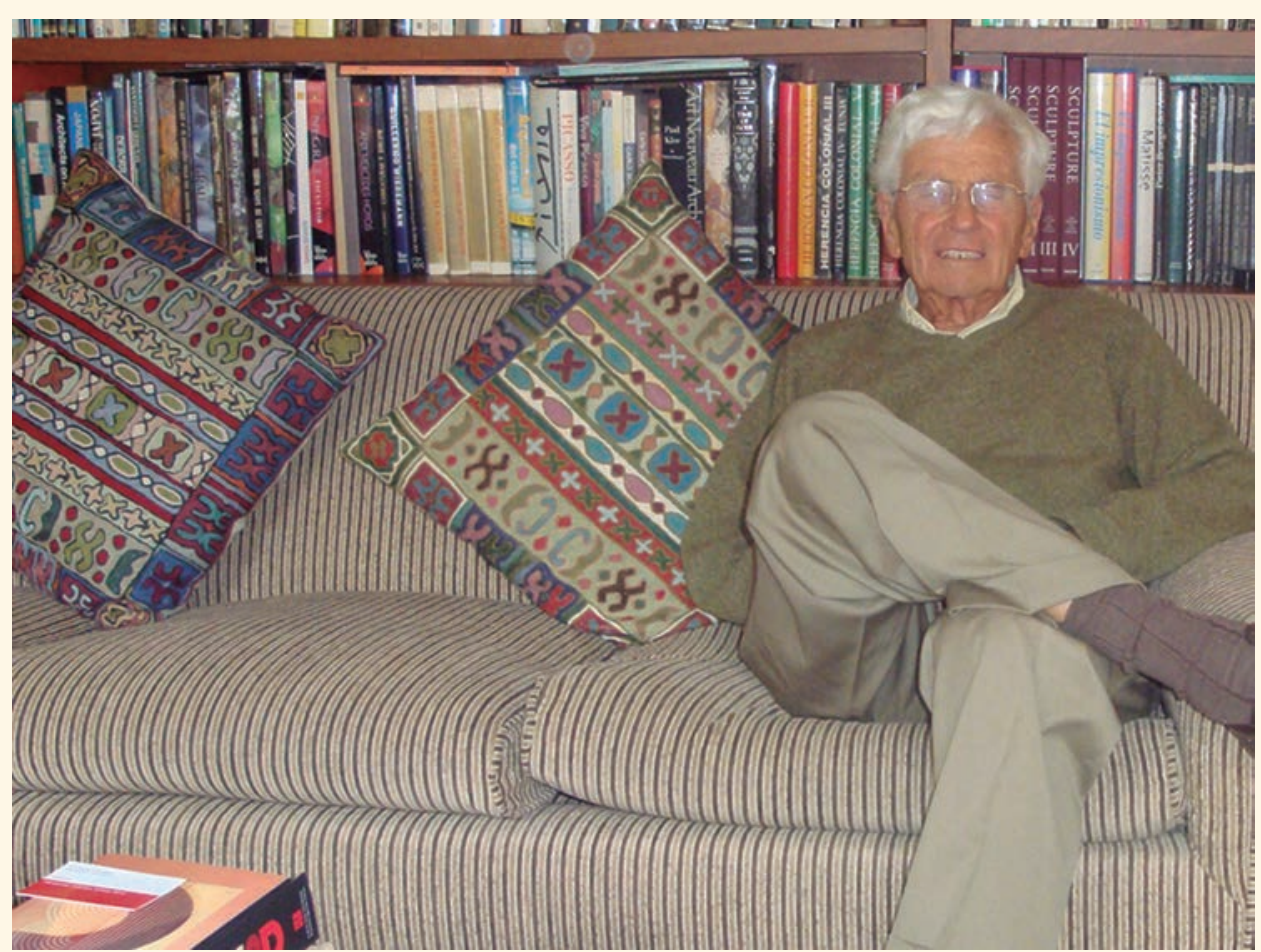

Llegar al apartamento del arquitecto Dicken Castro Duque, ubicado al Norte de la ciudad de Bogotá, es entrar en un universo donde se respira, además de la cordialidad y la bienvenida dada a los amigos de toda la vida, la experiencia de un maestro de la arquitectura de tipo integral que con gran sencillez y carisma comparte su visión del mundo y de nuestro oficio.

Esa tarde de sábado, inmersos y gratamente rodeados por una pequeña parte de su colección bibliográfica de 14.000 títulos, que como mudos testigos señalaban una gran riqueza de formación intelectual, conversamos con el arquitecto Dicken acerca de su vida, -su formación académica hecho de por sí fundamental en su concepción del ser y del hacer propios de la arquitectura y del diseño- $y$ de sus trabajos como parte del equipo trabajo conformado junto con los arquitectos Arturo Robledo Ocampo y Hans Drews Arango en la firma RDC Arquitectos.

Con su hablar sencillo y con actitud bastante modesta, este maestro de la arquitectura y del diseño gráfico del siglo $X X$ compartió su visión muy personal acerca de estos temas, se tuvo siempre como hilo conductor los referentes básicos de desarrollo del presente trabajo de investigación, hilo que algunas veces se disolvía y enriquecía a partir del traer a colación anécdotas, apreciaciones acerca del mundo, producto de sus innumerables viajes, y discusiones acerca de la concepción del ejercicio de la arquitectura llevada a cabo por parte de las nuevas generaciones de arquitectos en nuestro país. 
ONE INTERVIEW WITH ARCHITECT DICKEN CASTRO DUQUE

Jorge Alberto Villamizar Hernández *

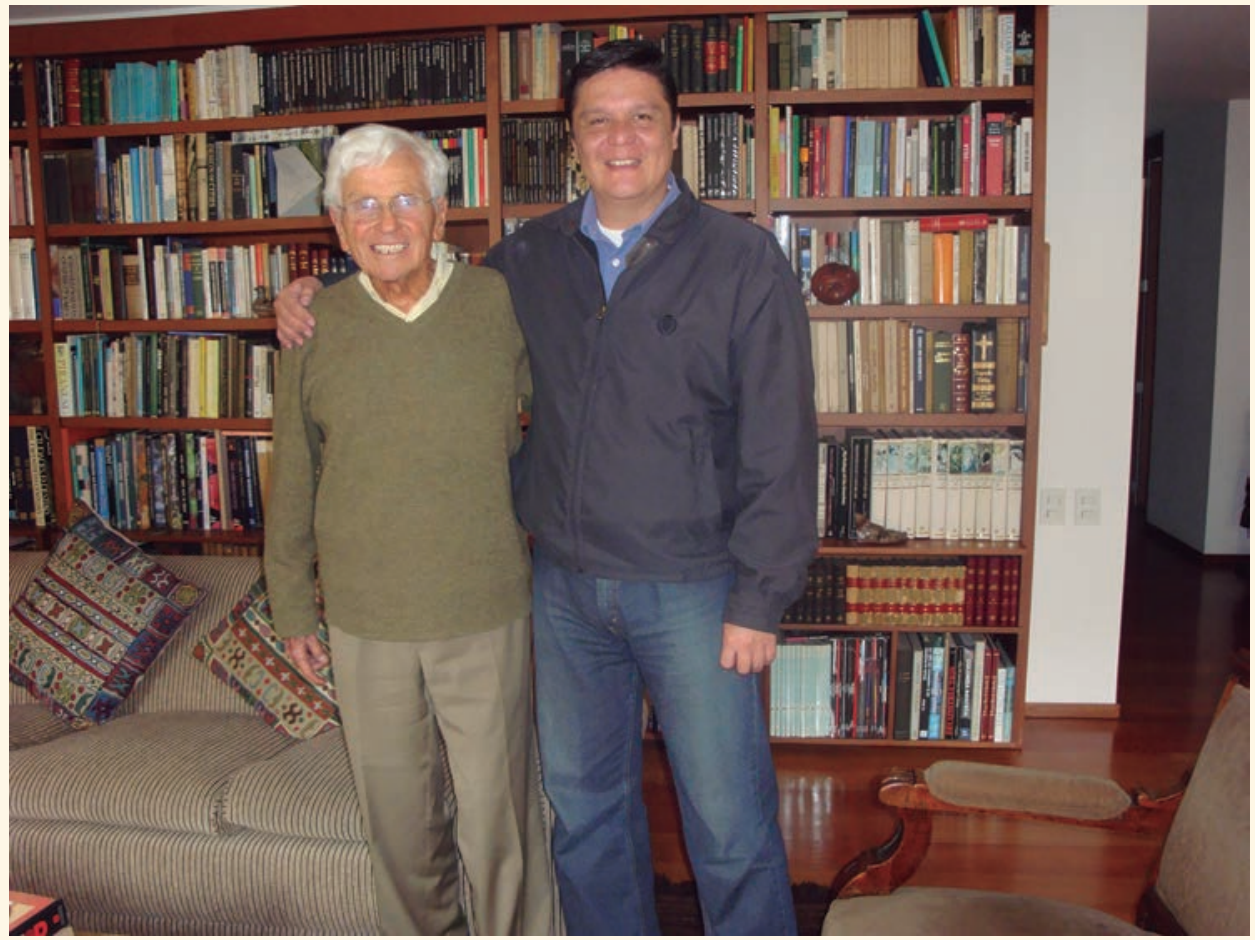

Para el arquitecto Dicken, un elemento fundamental que debe establecerse como punto de partida y de referencia del desarrollo de la arquitectura en Colombia es el hecho que la generación de arquitectos formados en nuestro país durante la década de los años 1940-1950 se constituye en la primera generación de arquitectos que llevó a cabo sus estudios aquí en Colombia en la mejor Facultad de Arquitectura existente: la Facultad de Arquitectura de la Universidad Nacional de Bogotá.

La condición fundamental que dio relevancia y destacó de manera particular a este proceso de formación profesional es que se tuvo la fortuna de contar con una planta docente conformada por arquitectos extranjeros llegados de Europa a raíz del éxodo generado por la II Guerra Mundial y, a su vez, por arquitectos colombianos formados en el extranjero, los cuales portaban ya como base de su quehacer profesional y formación intelectual la visión global del manejo del problema arquitectónico en aquel momento de la historia. La generación formada en esta década bajo estos referentes ha de destacarse posteriormente dentro del panorama general de la arquitectura en nuestro país por un brillante ejercicio profesional caracterizado por las propuestas innovadoras de las soluciones arquitectónicas en términos del contexto de la época en cuanto a la concepción cultural del proyecto arquitectónico en sí así como por la permanente, innovadora y enriquecedora reinterpretación de soluciones espaciales propias de nuestro país, sustentada esta última en gran parte por el manejo, tanto arquitectónico como técnico constructivo, de los materiales de cada región bajo el concepto y fundamento propio de los nuevos lenguajes derivados de los principios de la arquitectura moderna.
Arquitecto. Especialista en proyectos arquitectónicos Especialista en docencia universitaria. Docente investigador USTA Bucaramanga
Arquitectos Dicken Castro y Jorge Alberto Villamizar

Fuente: Jorge Alberto Villamizar Hernández 
Fotografía I. Dicken Castro

Fuente: Jorge Alberto Villamizar Hernández

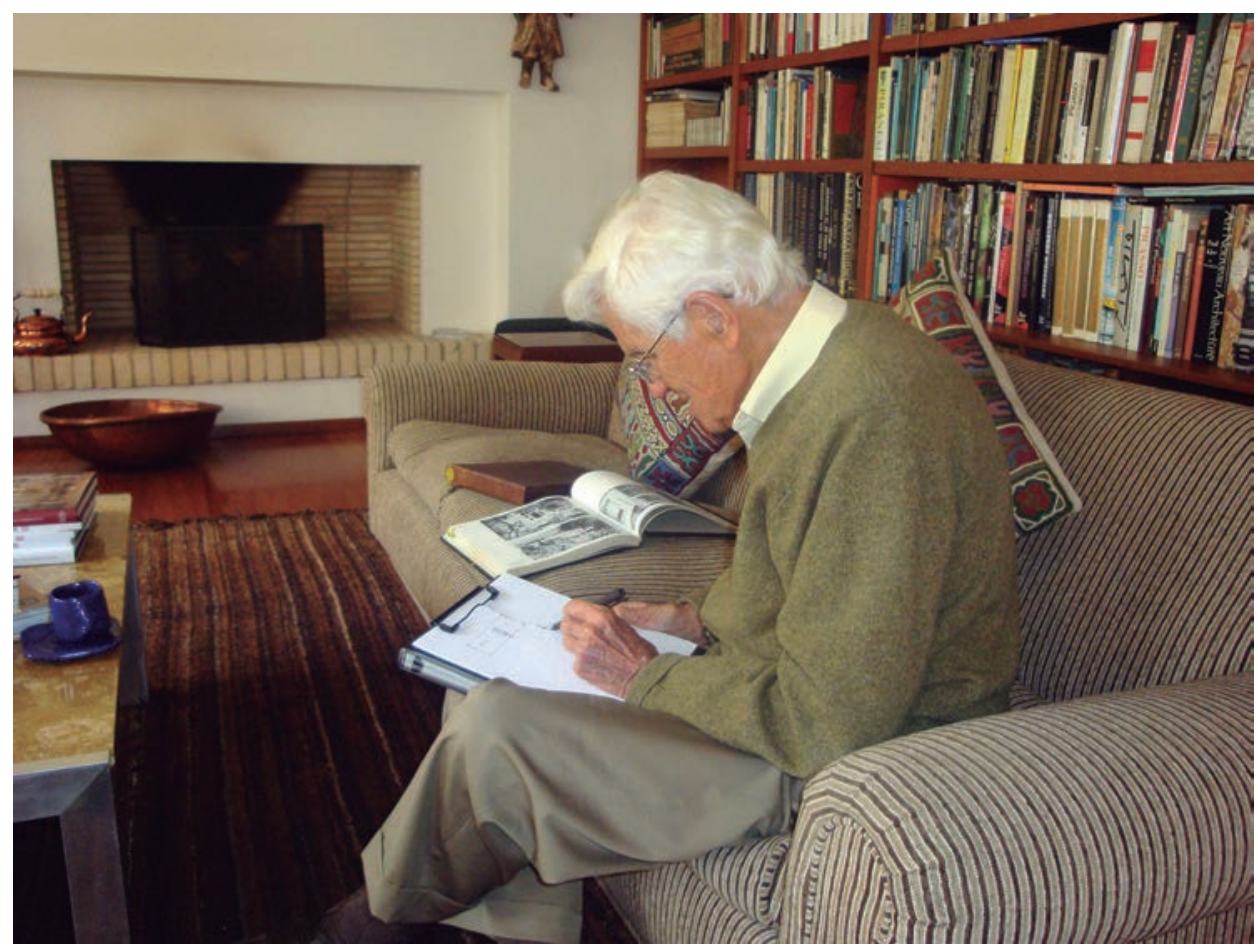

De igual manera esta generación se constituyó paralelamente en la primera generación de docentes colombianos formados en nuestro país, lo que establece un elemento para destacar de manera esencial dentro del panorama de la enseñanza y del ejercicio profesional: la adaptación a un medio conocido, autóctono, de los postulados de la arquitectura moderna.

Esta particularidad, unida a las condiciones propias del medio colombiano y del cambio sustancial de mentalidad en torno al concepto y manejo del problema arquitectónico planteado por la arquitectura moderna, establecieron una visión del problema arquitectónico y un manejo de la arquitectura en términos de enunciado, elaboración y puesta en marcha sólidamente influenciados y cimentados por los principios y postulados del movimiento moderno.

Durante su proceso de formación académica, el arquitecto Dicken Castro contó con docentes de la talla de los arquitectos Bruno Violi, Leopoldo Rother y Santiago de la Mora, quienes a partir del desarrollo propio de sus cátedras y en el manejo de sus proyectos arquitectónicos, a los cuales frecuentemente llevaban a sus estudiantes como parte de su proceso enseñanza aprendizaje, establecieron como referente clave de esta etapa un gran fundamento teórico como base de su enseñanza, el cual se encontraba basado específicamente en tres paradigmas fundamentales de la arquitectura moderna:

- Le Corbusier. Los cinco principios de Le Corbusier y la fundamentación básica de su obra fue absorbida en su totalidad y llegó a ser considerado dentro del ambiente académico como una especie de ídolo o figura de culto, cuya influencia que se vio reforzada por la visita llevada a cabo por este arquitecto a la ciudad de Bogotá en el año 1947 para llevar a cabo el diseño y el desarrollo del Plan Regulador de la ciudad.

- Mies Van Der Rohe. La influencia de los postulados de esta maestro alemán, posteriormente nacionalizado estadounidense, se evidenció de manera especial en la búsqueda constante al interior del ejercicio de diseño de una economía en el manejo de 
los materiales locales y de los elementos estructurantes del proyecto arquitectónico.

- La Bauhaus. La unidad de arte y artesanía para el logro de una construcción unitaria y la primacía de la concepción técnico constructiva reflejada de manera evidente en el objeto arquitectónico fueron los elementos tomados de los postulados de esta escuela alemana y marcaron de forma evidente los ejercicios de diseño desarrollados a lo largo del proceso de formación.

Estos paradigmas, considerados esenciales para llevar a cabo una buena nueva arquitectura, se complementaban con dos paradigmas más, no menos importantes: la obra del arquitecto finlandés Alvar Aalto y los trabajos del arquitecto holandés Gerrit Thomas Reitveld.

De la obra de Aalto se destacaba de manera especial su particular empleo en forma conjunta de un racionalismo funcionalista con el manejo de formas sinuosas, herramientas a través de las cuales lograba como resultado dentro del manejo espacial el acentuar la corporeidad del edificio; de los trabajos llevados a cabo por Reitveld se tomaron los postulados de su ejercicio arquitectónico y de diseño efectuados con la escuela De Stijl.

A partir de esta base conceptual y como punto de partida de los elementos definitorios que daban solución al proyecto arquitectónico se establecieron como parte del ejercicio de la obra arquitectónica de la firma RDC los siguientes criterios en los procesos de concepción, diseño, desarrollo y puesta en obra del proyecto arquitectónico:

- Criterio de economía. Este criterio se encontraba presente tanto en lo referente al manejo de la expresión arquitectónica como en el manejo y empleo de los materiales del proyecto traducido en la solución técnico-constructiva aplicada.

- Criterio de funcionalidad total. Este criterio era considerado en extremo importante ya que se establecía en cada ejercicio de diseño como el elemento básico constitutivo y fundamento del objeto arquitectónico.

- Criterio de rigurosidad y severidad. Este criterio era aplicado de forma integral en todo sentido en cada uno de los elementos presentes en el objeto arquitectónico, siendo en algunas ocasiones llevado a extremos en términos de manejo de áreas mínimas en la definición de los espacios arquitectónicos planteados.

A diferencia de esta generación, la generación posterior perteneciente a la década formada entre los años 1950 - 1960, estableció como referente de su ejercicio profesional otra serie de criterios arquitectónicos menos arraigados que la generación anterior a la que ya hemos hecho mención.

En cuanto al tema de los proyectos arquitectónicos llevados a cabo por el equipo de la firma RDC Arquitectos, el arquitecto Dicken señala que los mismos hacen evidente en términos espaciales la aplicación de estos postulados y criterios tanto a nivel de la totalidad del objeto como en el manejo del detalle, señaló de esta manera, una concepción integral en términos del proyecto arquitectónico como tal.

Destaca con gran aprecio el proyecto de Refugio y Teatro Infantil para el Club Los Lagartos de Bogotá, proyecto que explica detalladamente y toma para ello como referencia la reseña del mismo presente en el texto Forma Viva del cual toma un ejemplar de su biblioteca. 
Este proyecto se encuentra basado totalmente en la obra del arquitecto alemán Mies van Der Rohe y surge en el periodo posterior a la permanencia del arquitecto Dicken en Estados Unidos por un periodo de tres años y de las visitas y trabajos en la oficina del arquitecto alemán en los años 1960 y 1961.

El proyecto en su concepción espacial está estructurado a partir de tres zonas claramente definidas, que tiene como particularidad en el manejo del espacio dedicado a Sala Múltiple la influencia en la definición de las ventanas del empleo de una tipología particular utilizada habitualmente en las casas hacienda del territorio antioqueño y eje cafetero, las cuales eran accionadas para su abrir y cerrar por un sistema de pesas y poleas. Al levantarse las mismas el espacio de Sala Múltiple permite convertirse, al proyectarse y conectarse espacialmente con el espacio exterior, en un gran auditorio y escenario tanto cubierto como al aire libre.

El mural existente se desarrolló constructivamente a base de ladrillos que entran y salen de la superficie vertical y el referente usado se basa íntegramente en la propuesta del arquitecto Joseph Olbrich. La propuesta como tal resultó en extremo innovadora para la época, pues, en el periodo de los años 1957 a 1960 el ladrillo no era concebido ni se consideraba como un material de acabado que pudiera como tal dejarse a la vista asimilándose dentro del imaginario social y cultural a la condición de pobreza: "el ladrillo era siempre un material que había que esconder, cubrir, empañetar".

A las 4 de la tarde y luego de compartir con este maestro algunos de sus tesoros bibliográficos salgo de nuevo al frío de la capital con mis apuntes bajo el brazo y la necesidad, a partir de esta conversación, de precisar como inicio del texto del proyecto unos referentes acerca de la arquitectura moderna en el contexto latinoamericano y colombiano así como una contextualización inherente al proceso de formación del arquitecto en los años 1940 a 1960, tarea que con una sonrisa me ha señalado este gran amigo al decirme hasta pronto desde la puerta de su apartamento. 


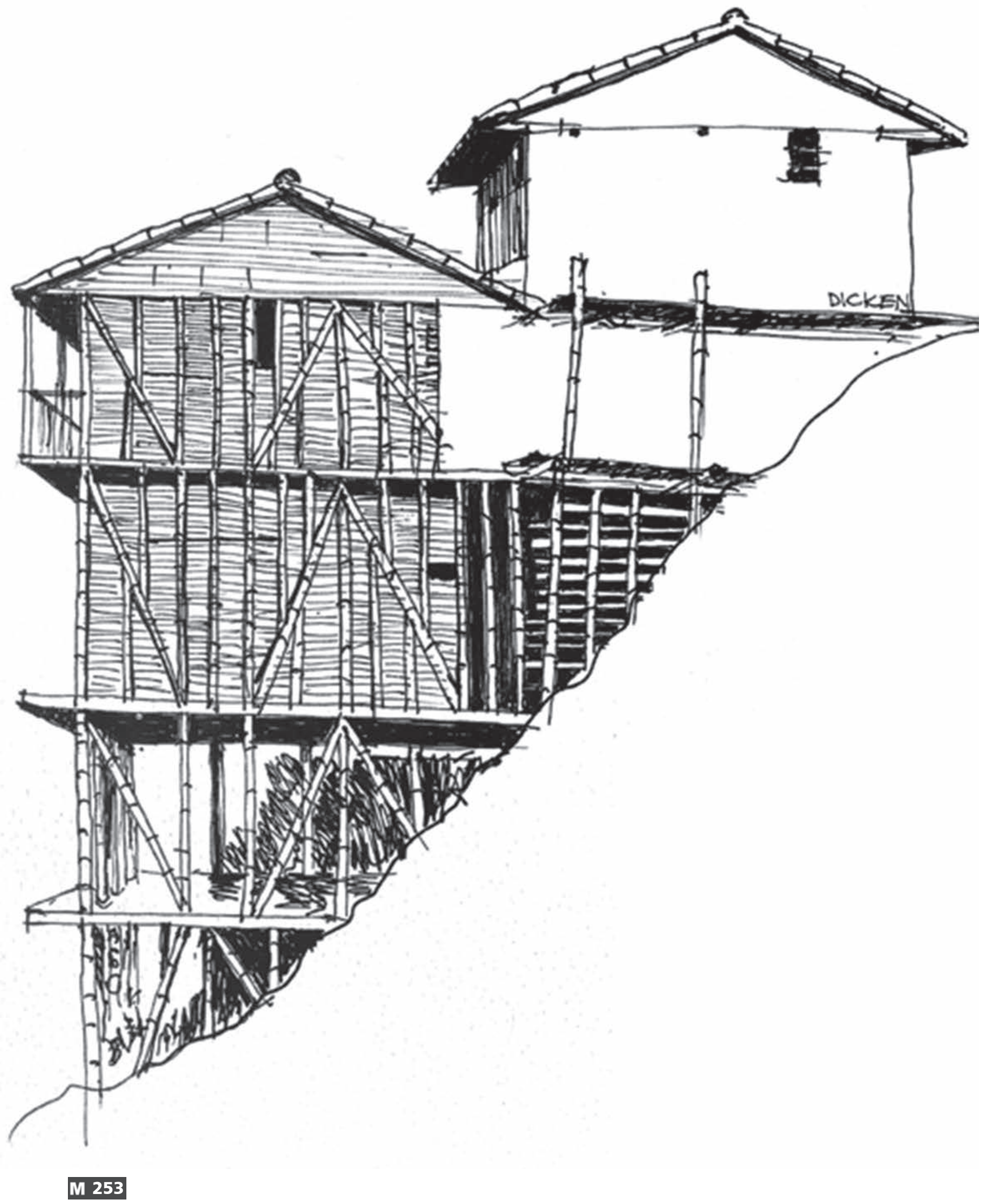

